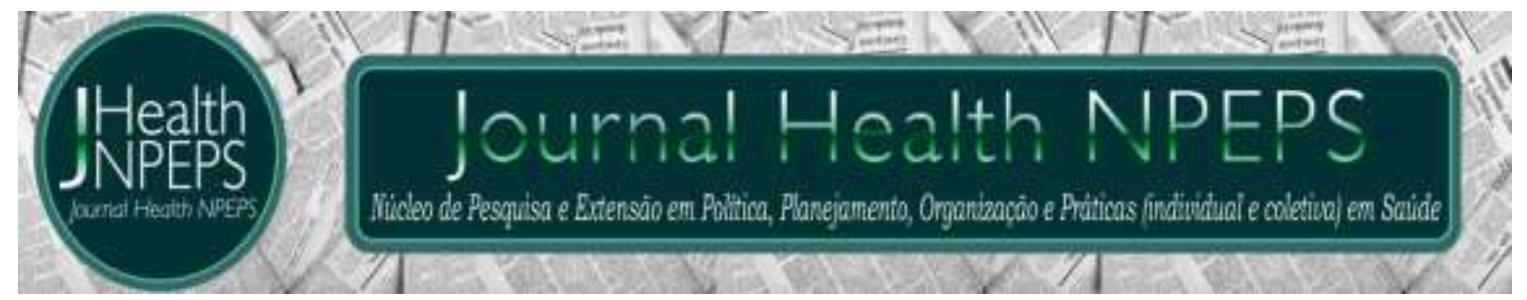

http://dx.doi.org/10.30681/252610104104

ARTIGO ORIGINAL

\title{
Interações medicamentosas entre psicofármacos e a relação com perfil de prescritores e usuários
}

\section{Drug interactions between psychoactive drugs and the relationship with prescribers and users profile}

\section{Interacciones farmacológicas entre drogas psicoactivas y la relación con los prescriptores y el perfil de los usuários}

\author{
Adilson Bosetto ${ }^{1}$, Claudinei Mesquita da Silva², Leyde Daiane de Peder ${ }^{3}$
}

\section{RESUMO}

Objetivo: traçar o perfil de prescritores e usuários de psicofármacos e verificar possíveis interações medicamentosas. Método: estudo retrospectivo e transversal. A amostra foi composta por prescrições de medicamentos sujeitos a controle especial, das listas C e B, dispensados no período de janeiro a março de 2017, para um total de 1344 pacientes atendidos em uma farmácia privada do município de Cascavel, Paraná. Resultados: as prescrições eram em sua maioria para o sexo feminino. Em relação à especialidade do prescritor, $423(31,5 \%)$ eram psiquiatras. As possíveis interações medicamentosas foram encontradas em 335 (24,9\%) pacientes, 206 $(61,5 \%)$ eram do tipo moderada e $129(38,5 \%)$ do tipo grave. Daqueles pacientes com interações potencialmente graves, 90 (70\%) utilizavam mais de um medicamento da mesma classe, no mesmo período. Em contrapartida, somente $12(5,83 \%)$ dos pacientes com interações moderadas tinham duplicidade terapêutica. Conclusão: o presente estudo identificou alta frequência de interações medicamentosas envolvendo psicofármacos e associação entre a duplicidade terapêutica e possíveis interações do tipo

\footnotetext{
${ }^{1}$ Farmacêutico. Mestre em Engenharia Agrícola. Farmacêutico da Cia Latinoamericana de Medicamentos. Cascavel, Paraná, Brasil. E-mail: adilson b19@hotmail.com ORCID ID: https://orcid.org/0000-0002-3985-7920

${ }^{2}$ Farmacêutico. Doutor em Ciências da Saúde. Docente do curso de Farmácia do Centro Universitário da Fundação Assis Gurgacz (FAG). Cascavel, Paraná, Brasil. E-mail: claudinei@fag.edu.br ORCID ID: https://orcid.org/0000-0003-4393-0331

${ }^{3}$ Farmacêutica. Doutora em Biociências e Fisiopatologia. Docente do curso de Farmácia do Centro Universitário da Fundação Assis Gurgacz (FAG). Cascavel, Paraná, Brasil. E-mail: leydepeder@yahoo.com.br ORCID ID: https://orcid.org/0000-0002-0814-2586 Autor principal Endereço para correspondência: Av. das Torres, 500 - Loteamento Fag, Cascavel - PR, 85806-095.
} 
grave, também, alta prevalência de interações graves em prescrições de psiquiatras e uso de psicotrópicos por mulheres.

Descritores: Interações de Medicamentos; Psicotrópicos; Prescrições de Medicamentos.

\section{ABSTRACT}

Objective: to profile prescribers and users of psychotropic drugs and verify possible drug interactions. Method: retrospective and cross-sectional study. The sample consisted of prescriptions for drugs subjected to special control, from lists $C$ and B, dispensed from January to March 2017 for a total of 1344 patients treated at a private pharmacy in the municipality of Cascavel, Paraná. Results: the prescriptions were mostly for females. Regarding the prescriber's specialty, 423 (31.5\%) were psychiatrists. Possible drug interactions were found in 335 (24.9\%) patients, of which 206 (61.5\%) were moderate and 129 (38.5\%) were severe. Of those patients with potentially serious interactions, 90 (70\%) used more than one medication in the same class, in the same period. In contrast, only 12 (5.83\%) of patients with moderate interactions had therapeutic duplicity. Conclusion: the present study identified a high frequency of drug interactions involving psychotropic drugs and an association between therapeutic duplication and possible serious type interactions, as well as a high prevalence of serious interactions in psychiatrist prescriptions and psychotropic use by women.

Descriptors: Drug Interactions; Psychotropic Drugs; Drug Prescriptions.

\section{RESUMEN}

Objetivo: Perfile a los prescriptores y usuarios de drogas psicotrópicas y verifique las posibles interacciones de drogas. Método: estudio retrospectivo y transversal. La muestra consistió en recetas de medicamentos sujetos a control especial, de las listas C y B, despachadas de enero a marzo de 2017, para un total de 1344 pacientes atendidos en una farmacia privada en Cascavel, Paraná. Resultados: las recetas fueron principalmente para mujeres. En cuanto a la especialidad de prescripción, 423 (31,5\%) eran psiquiatras. Se encontraron posibles interacciones farmacológicas en 335 (24,9\%) pacientes, 206 (61,5\%) fueron moderados y 129 (38,5\%) graves. De aquellos pacientes con interacciones potencialmente graves, 90 (70\%) usaron más de un medicamento en la misma clase durante el mismo período. En contraste, solo 12 (5,83\%) pacientes con interacciones moderadas tuvieron doble terapéutico. Conclusión: el presente estudio identificó una alta frecuencia de interacciones farmacológicas que involucran drogas psicotrópicas y una asociación entre la duplicación terapéutica y las posibles interacciones de tipo grave, así como una alta prevalencia de interacciones graves en las recetas de psiquiatras y el uso psicotrópico por parte de las mujeres.

Descriptores: Interacciones de Drogas; Psicotrópicos; Prescripciones de Medicamentos.

INTRODUÇÃO

0 aumento nas últimas décadas do número de medicamentos que agem a nível de 
sistema nervoso, também conhecidos como psicofármacos, ampliou as opções na farmacoterapia dos pacientes que sofrem de transtornos mentais. Em função disso, na prática clínica a polifarmácia vem se tornando cada vez mais frequente ${ }^{1}$. A polifarmácia psiquiátrica é entendida como o uso de dois ou mais psicofármacos, concomitantemente, no mesmo paciente ${ }^{2}$.

Nos Estados Unidos, a polifarmácia psiquiátrica mais que dobrou entre os anos de 2004 a 2013. Embora fosse mais comum em pacientes com diagnóstico de ansiedade, insônia ou depressão, essa prática teve aumento significativo em pacientes com queixa de dor, o que chamou a atenção neste período, indicando o aumento na prescrição de opióides $^{3}$. Em 2016, um estudo publicado naquele país revelou que um em cada seis adultos americanos relataram ter tomado psicofármacos pelo menos uma vez durante o ano de 2013. Este estudo identificou que $12 \%$ dos adultos relataram uso de antidepressivos; $8,3 \%$ usaram ansiolíticos, sedativos e hipnóticos; e 1,6\% antipsicóticos ${ }^{4}$.
No Brasil, o anuário estatístico do mercado farmacêutico de 2016 mostra que os medicamentos que atuam sobre o sistema nervoso central foram os que somaram maior faturamento naquele ano, eles representaram $14,6 \%$ da receita do setor regulado em termos de quantidade de embalagens vendidas. A representatividade desse grupo só não é maior do que de medicamentos destinados ao sistema cardiovascular ${ }^{5}$.

No tratamento psiquiátrico, associações medicamentosas podem ser benéficas quando proporcionam uma melhor eficácia terapêutica, entretanto, podem ser prejudiciais quando favorecem o aparecimento de reações adversas ou diminuição do efeito de um ou ambos os fármacos ${ }^{6}$.

Se por um lado o prescritor tem um rico arsenal farmacológico a seu dispor, por outro, o tratamento tornou-se mais perigoso para os pacientes em função da maior probabilidade de interações fármaco-fármaco. As interações medicamentosas correspondem a respostas farmacológicas cujos efeitos se originam pela utilização 
de um ou mais medicamentos, seja de forma simultânea, ou pela administração anterior de outros medicamentos ${ }^{7}$. Embora as interações medicamentosas com risco de vida sejam raras no âmbito ambulatorial, elas impactam na resposta às drogas e podem afetar os resultados em longo prazo. Estas alterações, nos aspectos farmacocinéticos farmacodinâmicos, podem interferir nos principais resultados que o paciente busca alcançar, como, eliminação dos sintomas, qualidade de vida e estabilização da doença ${ }^{8}$.

Interações medicamentosas que envolvem psicotrópicos comprometem principalmente 0 processo de metabolização. Elas podem aumentar consideravelmente 0 risco de intoxicação, devido à inibição de algumas isoenzimas do citocromo P450, e exacerbar as reações adversas 9 .

Estudos sobre o uso de medicamentos são úteis para promover o uso racional. Eles permitem conhecer o padrão de consumo de drogas em populações e avaliar se as necessidades de saúde daquela população estão sendo atendidas. Também auxiliam a identificar situações de risco e fundamentar ações e reflexões relacionadas à prescrição, dispensação e utilização de medicamentos $^{10}$.

Dessa forma, o objetivo deste trabalho foi traçar o perfil de prescritores e usuários de psicofármacos e verificar possíveis interações medicamentosas.

\section{MÉTODO}

Estudo retrospectivo e transversal, realizado em uma farmácia privada, a qual atende aproximadamente 4500 pessoas por mês e representa uma parcela importante de pacientes que compram medicamentos controlados no município de Cascavel, Paraná (PR), o qual possui 324.476 habitantes ${ }^{11}$. A fonte de dados do estudo foram prescrições armazenadas no estabelecimento. Incluiu-se os medicamentos prescritos, pertencentes à lista de medicamentos sujeitos ao controle especial pela Portaria 344/98 da ANVISA $^{12}$, dispensados entre janeiro 
e março de 2017, para 1344 pacientes. Como critérios de exclusão considerou-se prescrições ilegíveis, aquelas de uso veterinário e também as receitas de pacientes que fizeram aquisição de medicamentos em repetição durante o período do estudo ou trocaram a farmacoterapia durante o seu tratamento.

As informações contidas nas prescrições foram lidas em sua totalidade e considerou-se pertinente à pesquisa as seguintes variáveis: nome do paciente, sexo do paciente, número de medicamentos em uso, medicamentos prescritos, dose dos medicamentos, especialidade do prescritor. O nome do paciente foi considerado apenas durante a coleta de dados, a fim de garantir a fidelidade dos resultados obtidos, focando no paciente e não no número de prescrições analisadas. Dessa forma, pôde-se evidenciar a compra de medicamentos diferentes, durante 0 mesmo período, em receituários separados. Os dados foram armazenados no programa Microsoft Office Excel $2010 \AA$ e distribuídos no formato de tabelas. As potenciais interações

medicamentosas e o grau de severidade foram classificados utilizando-se o Micromedex ${ }^{\circledR}$ em: "Grave", quando requer intervenção médica para diminuir ou evitar efeitos adversos graves e pode apresentar perigo de vida; “Moderado", quando a interação pode resultar em uma exacerbação do problema de saúde do paciente; e "Menor", quando resulta em efeitos clínicos limitados. Neste trabalho, para o cálculo de porcentagem das interações foram consideradas apenas as de nível moderado e grave.

Os dados foram analisados pelo software estatístico XIStat versão 2016, realizando o teste de Qui Quadrado para Aderência, seguido do teste de acompanhamento de Marascuilo para a caracterização do perfil dos pacientes (sexo, especialidade médica, número de medicamentos), bem como para as possíveis interações observadas em níveis moderado e grave. Foi também realizado o cálculo de razão de chance - Odds Ratio (OR) para a ocorrência de interações medicamentosas em função dos 
prescritores de psicotrópicos,

período de estudo, 903 (67,2\%)

considerando como especialidade eram mulheres, sendo sua controle a Cardiologia.

Este estudo obteve frequência significativamente maior $(p=0,0001)$ do que a dos homens aprovação prévia do Comitê de $(\mathrm{n}=441 ; 32,8 \%)$. Os psiquiatras Ética em Pesquisa com Seres foram os médicos Humanos do Centro Universitário da Fundação Assis Gurgacz sob o parecer consubstanciado número 2.761.633 e CAAE: 91656718.3.0000.5219. significativamente mais relatados nas prescrições $\quad(n=423 ; 31,5 \%$; $\mathrm{p}<0,0001)$. Os pacientes que fizeram uso de apenas um medicamento correspondeu significativamente a maior RESULTADOS E DISCUSSÃO frequência $\quad(n=971 ; \quad 70,3 \%)$ $(p<0,0001)$, conforme Tabela 1.

Dos 1344 pacientes que utilizaram psicofármacos durante 0

Tabela 1 - Característica dos pacientes e das notificações de receitas de controle especial, aviadas em uma farmácia privada. Janeiro a março de 2017. Cascavel - PR, Brasil.

\begin{tabular}{lccc}
\hline Características & $\begin{array}{c}\text { Número de prescrições } \\
(\mathbf{n})\end{array}$ & $\begin{array}{c}\text { Frequência } \\
(\%)\end{array}$ & Valor de p \\
\hline Sexo do paciente & & & \\
$\quad$ Feminino & $903^{\mathrm{a}}$ & 67,2 & $<0,0001$ \\
$\quad \begin{array}{l}\text { Masculino } \\
\text { Especialidade médica que emitiu a receita }\end{array}$ & $441^{\mathrm{b}}$ & 32,8 & \\
$\quad$ Psiquiatra & $423^{\mathrm{a}}$ & 31,5 & $<0,0001$ \\
Clínico Geral & $278^{\mathrm{bf}}$ & 20,7 & \\
Neurologista & $123^{\mathrm{de}}$ & 9,2 & \\
Cardiologista & $100^{\mathrm{de}}$ & 7,4 & \\
Ortopedista & $94^{\mathrm{e}}$ & 7,0 & \\
Outras especialidades & $326^{\mathrm{f}}$ & 24,3 & \\
Número de medicamentos por paciente & & & \\
1 & $971^{\mathrm{a}}$ & 72,3 & $<0,0001$ \\
2 & $268^{\mathrm{b}}$ & 19,9 & \\
3 & $86^{\mathrm{c}}$ & 6,4 & \\
$\geq 4$ & $15^{\mathrm{d}}$ & 1,0 &
\end{tabular}

Observação: letras diferentes indicam as diferenças estatísticas entre as frequências das categorias das variáveis analisadas. Análise realizada pelo Teste de Marascuilo. Valor de P do teste de Qui Quadrado para Aderência.

Os resultados mostram uma relação significativa entre 0 consumo de drogas psicotrópicas e o sexo feminino. De acordo com a literatura, essa relação ocorre na maioria dos estudos sobre uso de 
psicofármacos ${ }^{13-15}$. Algumas

pesquisas sugeriram que as mulheres apresentam maior prevalência de transtornos mentais ${ }^{16}$. Os médicos consideram que as mulheres são mais frágeis, vulneráveis e com maior prevalência de transtornos afetivos. São mais propensas a revelar seus problemas emocionais ao médico e solicitar prescrições explicitamente. Além disso, elas mantêm visões mais positivas de drogas psicotrópicas e consultam com mais frequência do que os homens ${ }^{10,17}$.

Entende-se que as especialidades médicas mais habilitadas para acompanhar um paciente em uso de psicotrópicos sejam neurologistas e/ou psiquiatras. Neste trabalho, a porcentagem de pacientes que foram atendidos por estes profissionais soma $40,7 \%$ do total. Este número pode ser considerado bom devido a atual condição de assistência à saúde brasileira. Trabalhos similares encontrados na literatura, como o estudo de Balen et $a l^{18}$, realizado em uma farmácia básica, também no município de Cascavel (PR), relatou $33,5 \%$ de prescrições realizadas por neurologistas e/ou psiquiatras.

Os clínicos gerais são, em grande parte dos estudos nacionais sobre o tema, os médicos que mais prescrevem psicofármacos. Em um estudo realizado no Maranhão, apenas $17,9 \%$ das prescrições foram emitidas por psiquiatras ou neurologistas ${ }^{19}$.

Para a avaliação das possíveis interações entre os psicofármacos foram selecionados os 372 pacientes que utilizaram mais de um medicamento controlado naquele período. Destes, 3,2\% (12) pacientes não puderam ter seus medicamentos comparados pelo Micromedex ${ }^{\circledR}$ em função de limitações do software. Entre as possíveis interações, 55,4\% (206) foram interações moderadas e $34,7 \%$ (129) interações graves entre os medicamentos em uso.

No presente estudo, os psiquiatras foram os médicos responsáveis por $52,5 \%$ das prescrições contendo possíveis interações. Do total das receitas advindas desses profissionais, 41,6\% continham potencial interação, sendo que prescrições oriundas desta especialidade apresentaram 
2,8 vezes mais chances de advindas de cardiologistas. Os apresentarem interações dados estão descritos na Tabela 2.

medicamentosas do que prescrições

Tabela 2 - Interações verificadas entre medicamentos prescritos por médicos de diferentes especialidades e presentes em receitas aviadas em uma farmácia privada. Janeiro a março de 2017. Cascavel - PR, Brasil.

\begin{tabular}{|c|c|c|c|c|c|c|}
\hline \multirow[t]{2}{*}{$\begin{array}{c}\text { Especialidade } \\
\text { Médica }\end{array}$} & \multicolumn{3}{|c|}{$\begin{array}{l}\text { Pacientes com dois ou mais } \\
\text { medicamentos }\end{array}$} & \multirow[t]{2}{*}{$\begin{array}{l}\mathrm{PCl} \\
(\%)\end{array}$} & \multirow[t]{2}{*}{ OR (IC 95\%) } & \multirow[t]{2}{*}{$\begin{array}{l}\text { Valor } \\
p\end{array}$} \\
\hline & $\begin{array}{c}\text { Sem int/ Leve } \\
\mathrm{n}(\%)\end{array}$ & $\begin{array}{c}\text { Int } \\
\text { moderada } \\
\mathrm{n}(\%)\end{array}$ & $\begin{array}{c}\text { Int grave } \\
\text { n (\%) }\end{array}$ & & & \\
\hline Psiquiatra & $10(5,4)$ & $100(53,5)$ & $76(41,1)$ & 41,6 & $2,8(0,7-18,8)$ & 0,202 \\
\hline Neurologista & $2(5,1)$ & $31(79,5)$ & $6(15,4)$ & 30,9 & $0,7(0,1-5,6)$ & 0,725 \\
\hline Geriatra & $1(5,9)$ & $10(58,8)$ & $6(35,3)$ & 30,2 & $2,2(0,4-17,6)$ & 0,406 \\
\hline Clínico Geral & $7(9,7)$ & $35(48,6)$ & $30(41,7)$ & 21,2 & $2,9(0,7-19,8)$ & 0,204 \\
\hline Ortopedista & $0(0)$ & $10(71,4)$ & $4(28,6)$ & 14,9 & $1,6(0,2-13,8)$ & 0,634 \\
\hline Outros & $3(13,6)$ & $14(63,6)$ & $5(22,7)$ & 8,8 & $1,2(0,2-9,5)$ & 0,863 \\
\hline Cardiologista & $2(20)$ & $6(60)$ & $2(20)$ & 8,0 & 1 & - \\
\hline Total & 25 & 206 & 129 & - & & \\
\hline
\end{tabular}

Legenda: Int - interação; PCl - Porcentagem de prescrições de cada especialidade contendo interações medicamentosas; $\mathrm{n}$ - número de pacientes; OR - Odds Ratio.

Todas as demais especialidades, com exceção da neurologia, apresentaram maior chance de risco de ocorrência de interações medicamentosas em suas prescrições do que quando comparados à cardiologia. Contudo, vale ressaltar que nenhum dos cálculos de razão de chance foi considerado estatisticamente significativo $(p>0,05)$.

Foram encontradas ao todo, 122 tipos de possíveis interações moderadas e 217 de potenciais interações do tipo grave. Não houve prevalência de determinado tipo de interação em nenhuma das duas classes, não havendo, portanto, diferenças estatísticas entre as frequências das interações medicamentosas encontradas $(p>0,05)$.

Daqueles pacientes com interações potencialmente graves, 90 (70\%) utilizavam mais de um medicamento da mesma classe, no mesmo período. Em contrapartida, somente 12 (5,83\%) pacientes com interações moderadas tinham duplicidade terapêutica. Vale salientar que as possíveis interações moderadas compreenderam mais da metade de todas as interações detectadas, indicando que o uso concomitante de medicamentos de mesma classe 
pode ser um fator de risco para que

- paciente experimente uma interação medicamentosa

classificada como grave. Foi observado que, quase em sua totalidade, esses pacientes não aparentavam apresentar ajuste de dose entre os medicamentos prescritos.

Tabela 3 - Principais possíveis interações visualizadas em prescrições de pacientes que fazem uso de psicofármacos e aviadas em uma farmácia privada. Janeiro a março de 2017. Cascavel - PR, Brasil.

\begin{tabular}{|c|c|c|c|c|}
\hline \multicolumn{2}{|l|}{ Tipo de Interação } & $\begin{array}{c}\text { Número } \\
(\mathrm{n})\end{array}$ & $\begin{array}{c}\text { Frequência } \\
(\%)\end{array}$ & $\begin{array}{c}\text { Valor de } \\
\mathrm{p}^{*}\end{array}$ \\
\hline \multicolumn{5}{|c|}{ Potenciais interações do tipo moderado } \\
\hline Duloxetina & Pregabalina & 9 & 7,4 & \multirow{5}{*}{0,724} \\
\hline Sertralina & Zolpidem & 7 & 5,7 & \\
\hline Escitalopram & Zolpidem & 6 & 4,9 & \\
\hline Paroxetina & Zolpidem & 6 & 4,9 & \\
\hline Alprazolam & Zolpidem & 4 & 3,3 & \\
\hline \multicolumn{5}{|c|}{ Potenciais interações do tipo grave } \\
\hline Carbonato de lítio & Venlafaxina & 5 & 2,3 & \multirow{5}{*}{0,992} \\
\hline Escitalopram & Quetiapina & 5 & 2,3 & \\
\hline Bupropiona & Sertralina & 4 & 1,8 & \\
\hline Escitalopram & Mirtazapina & 4 & 1,8 & \\
\hline Sertralina & Venlafaxina & 4 & 1,8 & \\
\hline
\end{tabular}

*Valor de p: Teste de Qui Quadrado para aderência.

Os resultados mostram também que entre as interações do tipo moderado prevaleceram àquelas relacionadas ao aumento de efeitos secundários e/ou colaterais, como tontura, sonolência, confusão mental e dificuldade de concentração. 0 medicamento mais envolvido nesse tipo de interação foi o zolpidem, responsável por 29,5\% (36) do total.

Nos últimos anos, as prescrições para benzodiazepínicos diminuíram progressivamente devido à insatisfação com os efeitos adversos desses medicamentos. Isso levou ao desenvolvimento de um grupo estruturalmente similar de derivados não-benzodiazepínicos, incluindo a zopiclona e o zolpidem $^{20}$. 0 zolpidem é amplamente utilizado em vários países e é mais tolerado entre os pacientes que sofrem de insônia, mas nem por isso é um medicamento menos perigoso. Seu potencial de interação é por vezes menosprezado e os efeitos adversos quando associados a outras drogas preocupa farmacêuticos em todo o mundo. 
Um estudo multicêntrico realizou levantamento de intervenções realizadas por farmacêuticos clínicos, $45 \%$ dessas intervenções com idosos envolviam os medicamentos zolpidem ou zopiclona. Além disso, foram identificadas 709 interações medicamentosas ${ }^{21}$. Em outro estudo, também em ambiente hospitalar, $10 \%$ de todas as intervenções sobre medicamentos envolviam o zolpidem ${ }^{22}$.

A interação mais prevalente do tipo moderada foi entre a pregabalina e duloxetina. 0 metabolismo hepático da duloxetina e a inibição das isoenzimas $2 \mathrm{D} 6$ e $1 \mathrm{~A} 2$ do citocromo P-450 (CYP450) e seu alto nível de ligação às proteínas plasmáticas resultam em muitas interações com outros medicamentos ${ }^{23}$.

Os efeitos depressores do sistema nervoso central ou respiratório podem ser aumentados de maneira adicional ou sinérgica em pacientes que tomam vários medicamentos que causam esses efeitos, especialmente em pacientes idosos ou debilitados ${ }^{24}$. Em função disso, é essencial o acompanhamento médico destes pacientes. Isso reforça ainda mais o fato da necessidade de controle especial desses medicamentos.

As complicações advindas das potenciais interações do tipo grave encontradas durante a pesquisa estão relacionadas, de acordo com o Micromedex ${ }^{\circledR}$, principalmente ao aumento do risco de problemas cardíacos em pessoas predispostas, diminuição do limiar convulsivo, aumento do risco de intoxicação e risco de síndrome serotoninérgica.

Um dos medicamentos detectados com frequência entre as possíveis interações graves é a venlafaxina. Estudos demonstraram que este medicamento e o seu metabolito ativo são inibidores potentes e seletivos da recaptação neuronal de serotonina e norepinefrina e fracos inibidores da recaptação da dopamina ${ }^{25,26}$.

A síndrome serotoninérgica é uma reação adversa a medicamentos, causada pela ativação excessiva de receptores pós-sinápticos da serotonina. Ela afeta principalmente o sistema nervoso central e é caracterizada por um estado mental alterado, sinais de irritabilidade 
neuromuscular e instabilidade autonômica ${ }^{27}$.

A toxicidade da serotonina secundária à terapia medicamentosa, interação ou overdose são fenômenos crescentes em todo o mundo. Os sintomas podem ser severos, implicando em risco de morte ou podem ser leves e resolvidos com a interrupção do tratamento. Em geral, o tratamento é de suporte e de curta duração 28 .

A combinação de lítio e venlafaxina, encontrada com frequência durante a pesquisa, foi previamente descrita como causa da síndrome serotoninérgica. Sabese que, o lítio aumenta os níveis de serotonina no líquido cefalorraquidiano, induzindo a síntese desta neurotransmissor ${ }^{29}$. Outra prática frequente foi a associação entre a venlafaxina e a sertralina e a associação entre escitalopram e mirtazapina. Não foram encontrados estudos consistentes que evidenciem os efeitos destas interações na população, apesar do potencial risco de síndrome serotoninérgica. Há estudos relatando que essa associação é em geral, bem tolerada e apresenta melhoras no quadro clínico de pacientes deprimidos $^{30}$, o que sugere que as possíveis interações entre esses medicamentos não resultam em reações clinicamente importantes ${ }^{31}$.

Fato este que não parece ocorrer com a possível interação entre quetiapina e escitalopram. Apesar de haver estudos validando o seu uso no tratamento da depressão, existe uma relação entre o uso combinado destes dois fármacos e um aumento no intervalo de $\mathrm{QT}^{32-33}$. 0 intervalo $\mathrm{QT}$ é uma medida eletrocardiográfica de despolarização (onda Q) e repolarização ventricular (onda $\mathrm{T})^{34}$. 0 alongamento na repolarização cardíaca cria um ambiente eletrofisiológico que favorece 0 aparecimento de arritmias cardíacas que podem resultar em fibrilação ventricular levando a morte súbita ${ }^{35}$.

Apesar do risco na utilização concomitante destes medicamentos, um estudo epidemiológico em farmácias na Bélgica, mostrou que a interação medicamentosa mais encontrada foi a associação entre escitalopram e 
quetiapina. Para essa associação, os farmacêuticos daquele país propuseram uma intervenção em $69,5 \%$ dos casos. Entretanto, em $42 \%$, o prescritor considerou a proposta de intervenção desnecessária ${ }^{36}$.

Outra associação frequentemente encontrada foi entre a bupropiona e inibidores seletivos da receptação da serotinina (ISRS), onde o mais comum foi a sertralina. Embora haja estudo relatando a eficácia do uso concomitante destes medicamentos, uma interação farmacocinética entre bupropiona e sertralina foi comprovada em camundongos ${ }^{37-38}$. A bupropiona é exclusivamente metabolizada pela enzima CYP2B6, membro do citocromo $\mathrm{P} 450$, em seu principal metabólito ativo hidroxibupropiona ${ }^{39}$. No referido estudo, os camundongos prétratados com sertralina exibiram uma pequena elevação no metabolismo da bupropiona. Os pesquisadores constataram que na presença de sertralina, a bupropiona foi significativamente diminuída no cérebro dos animais enquanto a exposição à hidroxibupropiona no plasma aumentou significativamente. Também, a relação hidroxibupropiona-bupropiona plasmática foi aumentada em 27\% nos camundongos tratados com sertralina, o que é indicativo de aumento da atividade do CYP2B ${ }^{38}$.

O uso de bupropiona está associado a um risco aumentado de convulsões. Essa tese foi confirmada pelo estudo de Alper et $a l^{40}$, que revisaram dados dos estudos clínicos reguladores das Fases II e III da Food and Drug Administration, sobre várias drogas psicotrópicas. Apesar de estudos sugerirem um efeito antiepiléptico menor de antidepressivos como a sertralina ${ }^{41}$, a associação entre dois fármacos que são metabolizados pela mesma via pode resultar em aumento na concentração sérica de bupropiona e favorecer episódios convulsivos.

Outro problema encontrado com frequência durante a análise das possíveis interações foi $o$ aumento de toxicidade de algumas drogas quando em associações. A procura por atendimento médico em função de intoxicações medicamentosas é um problema 
preocupante e crescente em todo o mundo. A prevalência de consultas de emergência por eventos adversos a medicamentos nos Estados Unidos foi estimada em 4 por 1000 indivíduos em 2013 e 2014. As classes de drogas mais comuns foram anticoagulantes, antibióticos, agentes diabéticos e analgésicos opióides ${ }^{42}$.

O relatório anual de 2016 do Sistema Nacional de Dados Estatísticos da Associação Americana de Centros de Controle de Intoxicação aponta que os sedativos (compreendendo hipnóticos e antipsicóticos) e os antidepressivos estão entre as cinco principais classes de substância mais frequentemente envolvidas em todas as intoxicações atendidas pelo serviço de saúde americano. 0 documento revelou ainda que de todos os compostos que causaram intoxicação naquele país, os medicamentos sedativos foram os que aumentaram mais rapidamente, sendo em média $10,7 \%$ maior a cada ano. Nos últimos 15 anos estimou-se um aumento de 2088 casos de intoxicação por sedativos por ano ${ }^{43}$.
O escasso número de publicações acerca do uso de psicotrópicos e suas consequências no Brasil chama a atenção para um problema preocupante. Com dados epidemiológicos inconsistentes, há dificuldades na implantação de possíveis intervenções com o intuito de diminuir as ocorrências de efeitos adversos a medicamentos. A atenção farmacêutica e o acompanhamento

farmacoterapêutico podem auxiliar no sentido de preservar a saúde dessa população ${ }^{44}$.

Neste estudo, 90,8\% (304) das pessoas que utilizaram concomitantemente mais de um tipo de medicamento controlado no mesmo período, esteve vulnerável a experimentar pelo menos uma possível interação medicamentosa entre psicofármacos. Quando se considera todas as pessoas que utilizaram esses medicamentos naquele período, $25 \%$ da amostra (337) está na parcela da população com provável chance de experimentar uma interação medicamentosa do tipo fármacofármaco.

É preciso salientar que o uso de polifarmácia em transtornos 
psiquiátricos pode ser justificado em função do estado clínico do paciente em casos graves de doença. Os profissionais de saúde, especialistas ou não, devem estar cientes das possíveis interações medicamentosas, para que possam manejar possíveis eventos adversos relacionados às associações de risco. 0 acompanhamento farmacoterapêutico de pacientes polimedicados pode evitar problemas relacionados a essa prática.

Os dados acima apresentados devem ser analisados considerando algumas limitações, pois foram coletados em uma farmácia privada onde a maioria das prescrições advinha de consultas particulares. Dessa forma, o perfil de atendimento desses pacientes pode não refletir a situação de pacientes atendidos pelo sistema público de saúde. É preciso considerar também que as possíveis interações identificadas no estudo não foram investigadas clinicamente a fim de detectar um dano real ao paciente. Um futuro estudo com esse foco poderia ser realizado, na perspectiva de verificar a significância clínica dessas possíveis interações, bem como realizar um acompanhamento farmacoterapêutico de pacientes polimedicados com psicofármacos.

\section{CONCLUSÃO}

0 presente estudo demonstrou que a maioria das pessoas que utilizaram psicofármacos durante o período de estudo eram mulheres. Também identificou alta frequência de potenciais interações medicamentosas envolvendo psicofármacos. As principais interações potenciais do tipo moderadas e graves foram observadas. Verificou-se associação positiva entre a prescrição de fármacos da mesma classe e potenciais interações graves. Essas possíveis interações, deu-se a partir de prescrição realizadas em sua maioria por médicos psiquiatras.

\section{REFERÊNCIAS}

1. Cadogan CA, Ryan C, Hughes CM. Appropriate polypharmacy and medicine safety: when many is not too many. Drug Saf. 2016; 39(2):109-16. 
2. Medical Directors Council and State Medicaid Directors (NASMHPD). Alexandria, Virginia: 2001. National Association of State Mental Health Program Directors: Technical Report on Psychiatric Polypharmacy. Disponível em https://www.nasmhpd.org/sites /default/files/Polypharmacy.pdf . Acesso em 28 de julho de 2019.

3. Maust DT, Gerlach LB, Gibson A, Kales HC, Blow FC, Olfson M. Trends in central nervous system-sctive polypharmacy among older adults seen in outpatient care in the United States. JAMA Intern Med. 2017; 177(4):583-85.

4. Moore TJ, Mattison DR. Adult Utilization of Psychiatric Drugs and Differences by Sex, Age, and Race. JAMA Intern Med. 2017; 177(2):274-5.

5. Brasil. Ministério da Saúde. Agência Nacional de Vigilância Sanitária. Anuário Estatístico do Mercado Farmacêutico 2016. Disponível em: http: / / portal.anvisa.gov.br/docu ments/374947/3413536/Anu\%C3 \%A1rio+Estat\%C3\%ADstico+do+Me rcado+Farmac\%C3\%AAutico+- +2016/485ddf50-a37f-469f-89e529643c5c9df5. Acesso em: 28 de outubro de 2019.

6. Patel RI, Beckett RD. Evaluation of resources for analyzing drug interactions.

J Med Libr Assoc. 2016; 104(4):290-95.

7. Hanlon JT, Perera S, Newman $A B$, Thorpe JM, Donohue JM, Simonsick EM, et al. Potential drug-drug and drug-disease interactions in well-functioning community-dwelling older adults. J Clin Pharm Ther. 2017; 42(2):228-33.

8. English BA, Dortch M, Ereshefsky L, Jhee S. Clinically significant psychotropic drug-drug interactions in the primary care setting. Curr Psychiatry Rep. 2012; 14(4):376-90.

9. Silva SN, Lima MG, Ruas CM. Analysis of Potential Drug Interactions in Brazilian Mental Health Services: Prevalence and Associated Factors. J Young Pharm. 2018; 10(2):237-40.

10. Abi-Ackel MM, Lima-Costa MF, Castro-Costa E, Loyola Filho Al. Uso de psicofármacos entre idosos residentes em comunidade: prevalência e 
fatores associados. Rev Bras Epidemiol. 2017; 20(1):57-69.

11. IBGE. Instituto Brasileiro de Geografia e Estatística (IBGE). Estimativa Populacional 2018. Disponível em: https: //www.ibge.gov.br/cidad es-e-

estados.html?view=municipio Acesso em: 30 de agosto de 2018.

12. Brasil. Ministério da Saúde. Portaria 344 de 12 de maio de 1998. Aprova o Regulamento Técnico sobre substâncias e medicamentos sujeitos a controle especial. Publicada em DOU $\mathrm{n}^{\circ} 91$, de 15 de maio de 1998. Acesso em: 16 de outubro de 2019.

13. Boyd A, Van de Velde S, Vilagut $G$, de Graaf R, O'Neill S, Florescu S, et al. Gender differences in mental disorders and suicidality in Europe: Results from a large crosssectional population-based study. J Affect Disord. 2015; 173:245-54.

14. Campanha AM, Siu ER, Milhorança IA, Viana MC, Wang YP, Andrade LH. Use of psychotropic medications in São
Paulo Metropolitan Area, Brazil: pattern of healthcare provision to general population. Pharmacoepidemiol Drug Saf. 2015; 24(11):1207-14.

15. Estancial Fernandes CS, de Azevedo RCS, Goldbaum M, Barros MBA. Psychotropic use patterns: Are there differences between men and women? PLOS One. 2018; 13(11):e0207921.

16. Boyd A, Van de Velde S, Pivette $M$, Ten Have $M$, Florescu $S$, O'neill S, et al. Gender differences in psychotropic use across Europe: Results from a large cross-sectional, population-based study. Eur Psychiatry. 2015; 30(6):778-88.

17. Senicato C, Azevedo RCS, Barros MBA. Transtorno mental comum em mulheres adultas: identificando os segmentos mais vulneráveis. Cien Saude Colet. 2018; 23(8):2543-54.

18. Balen E, Giordani F, Cano MFF, Zonzini FHT, Klein KA, Vieira $\mathrm{MH}$, et al. Interações medicamentosas potenciais entre medicamentos psicotrópicos dispensados. J 
Bras Psiquiatr. 2017; 66(3):1727.

19. Torres MLD, Sousa LMG, Melo GC, Magalhães Júnior AA, Firmo WCA. Prescrição de psicotrópicos e especialidade médica: estudo em uma farmácia comercial no município do maranhão. Rev Cient ITPAC. 2014; 7(4).

20. Monti JM, Spence DW, Buttoo K, Pandi-Perumal SR. Zolpidem's use for insomnia. Asian $\mathrm{J}$ Psychiatr. 2017; 25:79-90.

21. Parent G, Rose FX, Bedouch P, Conorte $\mathrm{O}$, Charpiat B, Justeg $M$, et al. Interventions pharmaceutiques émises par les pharmaciens sur les médicaments psychotropes. L'Encéphale. 2015; 41(4):33945.

22. Arques-Armoiry E, Cabelguenne D, Stamm C, Janoly-Dumenil A, Grosset-Grange I, Vantard N, Maire $\mathrm{P}$, Charpiat B. Problèmes médicamenteux les plus fréquemment détectés par l'analyse

pharmacothérapeutique des prescriptions dans un centre hospitalier universitaire. Rev
Med Interne. 2010; 31(12):80411.

23. Ellis JJ, Sadosky AB, Ten Eyck LL, Cappelleri JC, Brown CR, Suehs BT, et al. Impact of potential pregabalin or duloxetine drug-drug interactions on health care costs and utilization among Medicare members with fibromyalgia. Clinicoecon Outcomes Res. 2014; 6:389-99.

24. Rodrigues MCS, Oliveira C. Interações medicamentosas e reações adversas a medicamentos em polifarmácia em idosos: uma revisão integrativa. Rev. Latino-Am. Enfermagem. 2016; 24: e2800.

25. Pfizer. Folheto Informativo EFFEXOR XR, Filadélfia, Estados Unidos, 2017. Disponível em: http: / / labeling.pfizer.com/Sho wLabeling.aspx?format=PDF\&id =100. Acesso: 02 nov. 2018.

26. Szkutnik-Fiedler D, Grabowski $T$, Balcerkiewicz $M$, Michalak $M$, Pilipczuk I, Wyrowski L, Urjasz $\mathrm{H}$, et al. The influence of $\mathrm{a}$ single and chronic administration of venlafaxine on tramadol pharmacokinetics 
in a rabbit model. Pharmacol Rep. 2017; 69(3):555-59.

27. Hillman AD, Witenko CJ, Sultan SM, Gala G. Serotonin syndrome caused by fentanyl and methadone in a burn injury. Pharmacotherapy. 2015; 35(1): 112-7.

28. Schult RF, Morris AJ, Picard L, Wiegand TJ. Citalopram overdose and severe serotonin syndrome in an intermediate metabolizing patient. Am J Emerg Med. 2019; 37(10):1993.e5-1993.e6.

29. Boyer E. Serotonin syndrome. Wolters Kluwer. UpToDate. Disponível em: http://www.uptodate.com/con tents/serotoninsyndrome?source=search_ result\&search=serotonin+syndro me\&selectedTitle=1\%7E150. Acesso em 29 de dezembro de 2019.

30. Shelton RC. Serotonin and Norepinephrine Reuptake Inhibitors.

Handb Exp Pharmacol. 2019; 250:145-80.

31. Bleakley S. Antidepressant drug interactions: evidence and clinical significance. Prog Neuro Psychiatry. 2016; 20(3):21-7.

32. Nothdurfter C, Schmotz C, Sarubin N, Baghai TC, Laenger A, Lieb $M$, et al. Effects of escitalopram/quetiapine combination therapy versus escitalopram monotherapy on hypothalamic-pituitary-adrenalaxis activity in relation to antidepressant effectiveness. J Psychiatry Res. 2014; 52:15-20.

33. Khan Q, Ismail M, Haider I, Khan F. Prevalence of QT interval prolonging drug-drug interactions (QT-DDIs) in psychiatry wards of tertiary care hospitals in Pakistan: a multicenter cross-sectional study. Int J Pharm. 2017; 39(6):1256-64.

34. QT interval and drug therapy. Drug and Therapeutics Bulletin, BMJ, i2732, Londres, 2016. Disponível em: https://www.bmj.com/content /353/bmj.i2732. Acesso em 04 de novembro de 2018.

35. Shah AJ, Hocini $M$, Denis $A$, Derval N, Sacher F, Jais $P$, et al. Polymorphic Ventricular Tachycardia/Ventricular Fibrillation and Sudden Cardiac 
Death in the Normal Heart. Card Electrophysiol Clin. 2016; 8(3):581-91.

36. Vandael E, De Wulf I, Foulon V. Drug-drug interactions with risk of QT-prolongation. A epidemiological study in Belgian community pharmacies. J Pharm Belg. 2016; (4):14-23.

37. Marshall RD, Johannet CM, Collins PY, Smith H, Kahn DA, Douglas CJ. Bupropion and sertraline combination treatment in refractory depression. J Psychopharmacol. 1995; 9(3):284-86.

38. Molnari JC, Hassan HE, Myers $A L$. Effects of sertraline on the pharmacokinetics of bupropion and its major metabolite, hydroxybupropion, in mice. Eur J Drug Metab Pharmacokinet. 2012; 37(1):57-63.

39. Kharasch ED, Crafford A. Common Polymorphisms of CYP2B6 Influence Stereoselective Bupropion Disposition. Clin Pharmacol Ther. 2019; 105(1):142-152.

40. Alper K, Schwartz KA, Kolts RL, Khan A. Seizure Incidence in Psychopharmacological Clinical Trials: An Analysis of Food and
Drug Administration (FDA) Summary Basis of Approval Reports. Biol Psychiatry. 2007; 62(4):345-54.

41. Rosenstein DL, Nelson JC, Jacobs SC. Seizures associated with antidepressants: A review. J Clin Psychiatry.1993; 54(8):289-99.

42. Shehab N, Lovegrove MC, Geller Al, Rose KO, Weidle NJ, Budnitz DS. US Emergency Department Visits for Outpatient Adverse Drug Events, 2013-2014. JAMA. 2016; 316(20):2115-25.

43. Gummin DD, Mowry JB, Spyker DA, Brooks DE, Fraser MO, Banner W. Annual report of the american association of poison control centers' national poison data system (NPDS): 34th Annual Report. Clin Toxicol. 2017; 55(10):1072-252.

44. Pereira MV, Alencar JS, Souto RP, Pinto NB, Saraiva SEM. Grau de conhecimento dos pacientes sobre o tratamento: estratégia para uso racional de medicamentos. J Health NPEPS. 2016; 1(1):31-39. 
Conflito de interesses: Os autores declaram não haver conflito de interesses.

\section{Participação dos autores:}

- Concepção: Bosetto A, Peder LD.

- Desenvolvimento: Bosetto A, Peder LD.

- Redação e revisão: Bosetto A, Silva CM.

Como citar este artigo: Bosetto A, Silva CM, Peder LD. Interações medicamentosas entre psicofármacos e a relação com perfil de prescritores e usuários. J Health NPEPS. 2020; 5(1):187-206.

Submissão: 18/10/2019

Aceito: $06 / 01 / 2020$

Publicado: 01/06/2020 